\title{
Dasatinib enhances antitumor activity of paclitaxel in ovarian cancer through Src signaling
}

\author{
JUAN XIAO $^{1 *}$, MANMAN XU $^{2 *}$, TENG HOU $^{1}$, YONGWEN HUANG $^{1}, \mathrm{CHENLU} \mathrm{YANG}^{1}$ and $\mathrm{JUNDONG} \mathrm{LI}^{1,3}$ \\ ${ }^{1}$ Department of Gynecology, Sun Yat-sen University Cancer Center, State Key Laboratory of Oncology in South China, \\ Collaborative Innovation Center for Cancer Medicine, Guangzhou, Guangdong 510060; ${ }^{2}$ Department of Obstetrics and \\ Gynecology, The First Affiliated Hospital, Sun Yat-sen University, Guangzhou, Guangdong 510080; ${ }^{3}$ Department of \\ Gynecology, Affiliated Tumor Hospital, Xinjiang Medical University, Ürümqi, Xinjiang 830000, P.R. China
}

Received March 5, 2014; Accepted February 17, 2015

DOI: $10.3892 / \mathrm{mmr} .2015 .3784$

\begin{abstract}
Src family tyrosine kinase (SFK) activation is associated with ovarian cancer progression. Therefore, SFKs are targets for the development of potential treatments of ovarian cancer. Dasatinib is a tyrosine kinase inhibitor that targets SFK activity, and is used for the treatment of B cell and Abelson lymphomas. At the present time, the potential effect of dasatinib on ovarian cancer is not clear. The aim of the present study was to investigate the antitumor activity of dasatinib, alone and in combination with paclitaxel, in ovarian cancer in vitro and in vivo. In the present study, the expression of Src and phospho-Src-Y416 (p-Src) was measured in six ovarian cancer cell lines using western blotting and immunohistochemistry. In addition, cell viability and apoptosis were measured using an MTT assay and annexin V-fluorescein isothiocyanate staining. An ovarian cancer murine xenograft model was established, in order to evaluate the antitumor effect of dasatinib alone and in combination with paclitaxel in ovarian cancer. High levels of $\mathrm{p}$-Src protein expression were observed in all cell lines, as compared with healthy cells, which indicated activation of the Src signaling pathway. p-Src expression increased in ovarian cancer cells following paclitaxel treatment. Dasatinib treatment demonstrated anti-ovarian cancer properties, by downregulating p-Src expression and by inducing cancer cell apoptosis. Combined treatment with dasatinib and paclitaxel markedly inhibited proliferation and promoted apoptosis of ovarian cancer cells, compared with control cells. Combined dasatinib and paclitaxel treatment exhibited antitumor activities in vivo
\end{abstract}

Correspondence to: Mr. Jundong Li, Department of Gynecology, Sun Yat-sen University Cancer Center, State Key Laboratory of Oncology in South China, Collaborative Innovation Center for Cancer Medicine, 651 E, Dongfeng Ave, Guangzhou, Guangdong 510060, P.R. China E-mail: lijd@sysucc.org.cn

${ }^{*}$ Contributed equally

Key words: Src, Src family tyrosine kinase, dasatinib, paclitaxel, ovarian cancer and in vitro (combination indices, $0.25-0.93$ and $0.31-0.75$; and tumor growth inhibitory rates, $76.7 \%$ and $58.5 \%$, in A2780 and HO8910 cell lines, respectively), compared with paclitaxel treatment alone. Dasatinib monotherapy demonstrated anti-ovarian cancer activities. The effects of dasatinib and paclitaxel treatments on ovarian cancer cells appeared to be mediated by the Src pathway.

\section{Introduction}

Platinum and taxane-based chemotherapy following cytoreductive surgery are the predominant approaches for the treatment of advanced ovarian cancer (1). Although the majority of patients respond well to primary chemotherapy, $75 \%$ will experience a relapse and acquire drug-resistance (1). Novel therapeutic strategies are, therefore, required in order to improve the prognosis for such patients (2). Dasatinib is one such therapy that targets the breakpoint cluster region-Abelson (BCR-ABL) fusion protein and the Src family tyrosine kinases (SFKs) (3). SFKs consist of nine non-receptor tyrosine kinases that share a similar structure (4). Cellular-Src kinase (c-Src) is expressed in a number of types of cells. It is involved in numerous signaling pathways, and in the formation and development of tumors (5). Overexpression and activation of c-Src has been observed in the development of certain solid tumors, such as breast, colon, prostate, ovarian, pancreatic and non-small cell lung cancer, and squamous cell carcinoma of the head and neck (6-10). Budde et al (11) detected Src expression in 60 human tumor cell lines and demonstrated that ovarian cancer cell lines exhibited a moderate level of Src expression, compared with healthy cell lines. A further study demonstrated Src overexpression and activation in advanced-stage ovarian tumor cells (12). Similarly, c-Src and phospho-Src-Y416 (p-Src; Tyr416) were shown to be overexpressed in human ovarian cancer cells (13). A number of different Src inhibitors have been analyzed in certain tumors. Dasatinib is a multi-targeted inhibitor of the receptor tyrosine kinases, Src and the BCR-ABL fusion protein (14). In June 2006, the Food and Drug Administration approved the use of dasatinib for the treatment of imatinib-resistant or imatinib-intolerant patients with chronic myeloid leukemia, and for the treatment of patients with Philadelphia-chromosome-positive acute 
lymphoblastic leukemia, who may be resistant or intolerant to first-line treatments (15). Dasatinib therapy has been investigated in other types of malignancies and the results observed for the treatment of solid tumors are encouraging. A number of studies have confirmed the effectiveness of dasatinib treatment for solid tumors (16-21), although few studies have focused on ovarian cancer. Therefore, the effects of dasatinib on ovarian cancer remain unclear. Konecny et al (22) examined the effects of dasatinib in 34 human ovarian cancer cell lines and demonstrated that $24 / 34(71 \%)$ of representative ovarian cancer cell lines were highly sensitive to dasatinib. Furthermore, additive and synergistic interactions were observed, following treatment with dasatinib and carboplatin or paclitaxel. Similar results were presented by Teoh et al (23). However, the precise mechanisms underlying the antitumor effects of, and the interactions between, dasatinib and paclitaxel, such as cell survival, proliferation, autophagy, microtubule stability, motility and tumor angiogenesis remain unknown. The aim of the present study was to evaluate the antitumor properties of dasatinib alone and in combination with paclitaxel in ovarian cancer in vitro and in vivo. The mechanisms underlying the interactions between dasatinib and paclitaxel were also investigated.

\section{Materials and methods}

Drugs and reagents. In order to conduct an in vitro study, dasatinib (Selleck Chemicals, Houston, TX, USA) was dissolved in dimethylsulfoxide (DMSO; DaMao Chemical Reagent Factory, Tiangjin, China) at $10 \mathrm{mmol} / 1$ and stored at $-20^{\circ} \mathrm{C}$. Frequent freeze-thawing was avoided. In order to conduct an in vivo study, dasatinib was diluted in sterile distilled water at $1 \mathrm{mg} / \mathrm{ml}$, and stored at $4^{\circ} \mathrm{C}$ for $<7$ days. Paclitaxel (Bristol-Myers Squibb, New York City, NY, USA) was diluted in $3 \mathrm{mg} / \mathrm{ml}$ sterile distilled water. The rabbit polyclonal anti-Src (cat. no. 2108S; 1:100) and rabbit polyclonal anti-phosphorylated Src (cat. no. 2101S; 1:60) antibodies were purchased from Cell Signaling Technology, Inc. (Danvers, MA, USA). The monoclonal mouse GAPDH antibody (cat. no KC-5G5; 1:1,000) was purchased from Kangchen (Shanghai, China). The goat-anti-rabbit secondary (cat. no. sc-2054; 1:1,000), and goat-anti-mouse secondary (cat. no. sc-2005; 1:1,000) antibodies were obtained from Santa Cruz Biotechnology, Inc. (Santa Cruz, CA, USA). A horseradish peroxidase (HRP) polymer-conjugated anti-rabbit secondary antibody (cat. no. PV-6001; 1:1,000) was purchased from ZSGB-BIO (Beijing, China), and Annexin V-fluorescein isothiocyanate (FITC) was obtained from Merck Millipore (Darmstadt, Germany). An Apoptosis Detection kit was purchased from EMD Millipore (Billerica, MA, USA). DMSO, MTT, and polyvinylidene difluoride (PVDF) membranes were purchased from Sigma-Aldrich (St. Louis, MO, USA).

Cell lines and cell culture. The following six human ovarian cancer cell lines were used for analysis: A2780, HO8910, OVCAR3, CAOV3 and COC1 (Collection Conservation Center of Wuhan University, Wuhan, China), and SKOV3 (State Key Laboratory of Oncology in South China, Guangzhou, China). All cell lines were cultured in Dulbecco's modified Eagle's medium (DMEM; Thermo Fisher Scientific, Waltham, MA, USA), supplemented with $5 \%$ heat-inactivated fetal bovine serum (Guangzhou Ruite Bio-tec Co., Ltd., Guangzhou, China), penicillin $(50 \mathrm{U} / \mathrm{ml})$, and streptomycin $(50 \mu \mathrm{g} / \mathrm{ml})$ (North China Pharmaceutical Co., Ltd., Shijiazhuang, China). Cells were grown in a humidified incubator with $5 \% \mathrm{CO}_{2}$, at $37^{\circ} \mathrm{C}$.

Western blot analysis. Cells were cultured in six-well plates for $24 \mathrm{~h}$ and exposed to treatments when in the logarithmic growth phase. Cells were harvested and total protein was isolated. Total protein (20-40 $\mu \mathrm{g})$ was separated using electrophoresis, electrotransferred to PVDF membranes and then probed with the primary antibodies in Tris-buffered saline with Tween- $20^{\circledR}$ (TBST; Bioeasy Co., Ltd., Shenzhen, China)-5\% milk (1:1,000) overnight, at $4{ }^{\circ} \mathrm{C}$. The membranes were incubated with a peroxidase-conjugated secondary antibody in TBST-5\% milk $(1: 3,000)$ for $1-2 \mathrm{~h}$, at room temperature. Protein detection was achieved using enhanced chemiluminescence reagent and XAR film (Kodak, Rochester, NY, USA) according to the manufacturer's instructions. Anti-GAPDH was used as a positive control (24).

MTT assay. In order to measure cell viability and activity, cells in the logarithmic growth phase were seeded in 96-well plates (3,000 cells/well) and then treated with different drug concentrations. Concentrations were selected according to references $(16,25,26)$ and preliminary experiments, which demonstrated that dasatinib $(10 \mathrm{mg} / \mathrm{kg})$ and paclitaxel $(15 \mathrm{mg} / \mathrm{kg})$ had marked anti-ovarian cancer effects. The following concentrations were used: Dasatinib $(0.3125,0.625,1.25,2.5,5$, 10 and $20 \mu \mathrm{mol} / \mathrm{l})$, paclitaxel $(1.25,2.5,5,10$ and $20 \mu \mathrm{mol} / \mathrm{l})$ or a combination of dasatinib and paclitaxel $(250: 1$ or $500: 1)$. Negative control cells were treated with DMSO. Experiments were repeated three times. Following $72 \mathrm{~h}$ of drug treatment, $10 \mu \mathrm{l}$ of $5 \mathrm{mg} / \mathrm{ml} \mathrm{MTT}$ was added to each well for an additional $4 \mathrm{~h}$ of incubation at $37^{\circ} \mathrm{C}$, in $5 \% \mathrm{CO}_{2}$. The medium was subsequently removed and $100 \mu \mathrm{l}$ DMSO was added to the cells. Plates were agitated gently for $10 \mathrm{~min}$ at $37^{\circ} \mathrm{C}$ and absorbance at $570 \mathrm{~nm}$ was measured using a microplate reader (Molecular Devices, West Berkshire, UK). Cytotoxicity was evaluated by calculating the $50 \%$ inhibitory concentration $\left(\mathrm{IC}_{50}\right)$, and the additive or synergistic effects were assessed using combination indices (CI). $\mathrm{CI}<1$ indicated synergy, $\mathrm{CI}=1$ indicated additivity, and $\mathrm{CI}>1$ indicated antagonism (24), calculated using Calcusyn 2.0 software (Biosoft, Cambridge, UK).

Apoptosis assays. A2780 and HO8910 cell lines were cultured in six-well plates and treated with paclitaxel with or without dasatinib for $48-72 \mathrm{~h}$ in culture medium $\left(37^{\circ} \mathrm{C}, 5 \% \mathrm{CO}_{2}\right)$. The cells were stained using an Annexin V-FITC Apoptosis Detection kit. Subsequently, cell cycle and apoptosis were measured at $488 \mathrm{~nm}$, using flow cytometry (Beckman Coulter, Brea, CA, USA). Data were recorded as the percentage of cells in each phase of the cell cycle.

Tumor xenografts. A total of 87 female BALB/c nude mice age, 5-6 weeks old, weight, 18-20 g, were obtained from Hunan SJA Lab Animal Co. Inc. (Hunan, China) and were maintained in specific pathogen-free conditions. Experiments were approved by the animal care committee of Sun Yat-sen University (Guangzhou, China) and were performed under sterile conditions. Mice were housed in Sun Yat-sen University 


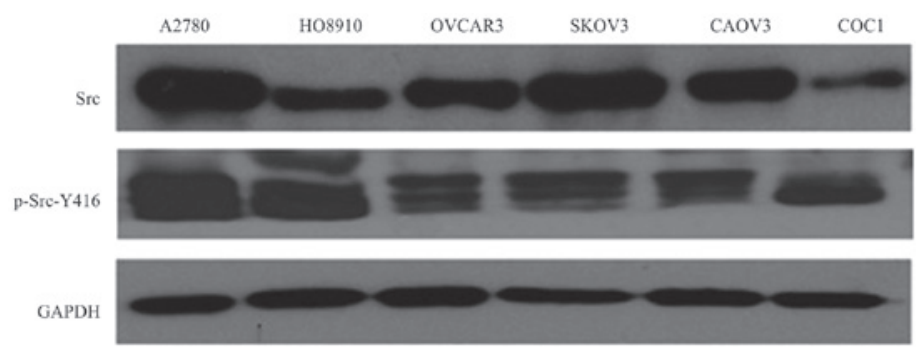

Figure 1. Expression of Src and p-Src (Tyr 416) in A2780, HO8910, OVCAR3, SKOV3, CAOV3, and COC1 type cell lines. p-Src, phospho-Src-Y416.

Medical Animal Center and maintained in a $12 \mathrm{~h}$ light/dark cycle with access to food and water. A2780 or HO8910 cell lines $\left(5 \times 10^{6}\right.$ cells) were subcutaneously injected into both side flanks, using a 23-gauge needle (each cell line was administered to two mice; Sun Yat-sun University Cancer Center). Once the tumors had reached a mean diameter of 8-10 $\mathrm{mm}$, the mice were sacrificed and the tumor tissue was cut into 1-2-mm fragments in serum-free DMEM, at $4^{\circ} \mathrm{C}$. The tumor fragments were subsequently transplanted subcutaneously into the flanks of recipient mice (42 mice were injected with the A2780 tumor fragments; 43 mice were injected with the HO8910 tumor fragments). The whole transplantation procedure was completed within 30-40 min. Tumor size and animal weight were monitored every 2-3 days. Once tumor volumes had reached 50-80 $\mathrm{mm}^{3}$ (mean diameter 3-5 mm, following 7 days of inoculation), mice were randomly divided into four groups of six, and treated intraperitoneally with one of the following: Dasatinib only (10 mg/kg, five times a week); paclitaxel only (15 mg/kg, once a week); combined dasatinib $(10 \mathrm{mg} / \mathrm{kg})$ and paclitaxel $(15 \mathrm{mg} / \mathrm{kg})$; or negative control (sterile distilled water). All treatments were administered for 4 weeks; dosage was derived from references $(16,25,26)$ and preliminary experiments, which demonstrated that dasatinib $(10 \mathrm{mg} / \mathrm{kg})$ and paclitaxel $(15 \mathrm{mg} / \mathrm{kg})$ had marked anti-ovarian cancer effects. Tumor volumes were calculated using the formula: volume $\left(\mathrm{mm}^{2}\right)=0.5 \mathrm{x}$ tumor length $\mathrm{x}$ tumor width ${ }^{2}$. Mice were sacrificed by cervical dislocation and tumor size, tumor weight, and mouse body weight were measured. Tumor tissues were excised and tissue samples were divided: One section was fixed in formalin (Guangzhou Chemical Reagent Factory, Guangzhou, China) for immunohistochemistry analysis and a second section was fixed in liquid nitrogen for western blotting. Tumor growth inhibitory rate (TIR) was calculated as follows: (average tumor weight in control group - average tumor weight in treatment group)/aver average tumor weight in control group x $100 \%$.

Immunohistochemistry. The xenograft tumor tissues were fixed in formalin, embedded in paraffin, sliced into 4- $\mu \mathrm{m}$ sections, and rehydrated using a graded alcohol series. Endogenous peroxidase activity was blocked using 3\% hydrogen peroxide (Guangzhou Chemical Reagent Factory) in methanol, for $10 \mathrm{~min}$. For antigen retrieval, slides were heated in a pressure cooker in 10 mM citrate buffer ( $\mathrm{pH}$ 6.0; Guangzhou Chemical Reagent Factory), for $10 \mathrm{~min}$. The slides were incubated with anti-Src antibody (1:100) or anti-P-Src antibody (1:60) overnight, at $4^{\circ} \mathrm{C}$. Slides were then incubated with an HRP polymer-conjugated anti-rabbit secondary antibody for $30 \mathrm{~min}$, at $37^{\circ} \mathrm{C}$ and the color was developed using diaminobenzidine for $5 \mathrm{~min}$. The nucleus was counter-stained using Meyer's hematoxylin (Guangzhou Chemical Reagent Factory). The negative control was obtained by replacing the primary antibody with normal rabbit immunoglobulin G. Human epithelial ovarian cancer slides were used as positive controls. Tumor tissue, fixed in liquid nitrogen, was pulverized in cell lysis buffer $(1,000 \mu \mathrm{l}$; Guangzhou Chemical Reagent Factory), on ice. Immediately, $1 \mathrm{mmol} / \mathrm{l}$ phenylmethanesulfonylfluoride (Guangzhou Chemical Reagent Factory) was added and the lysate was clarified twice by centrifugation at $12,000 \mathrm{x} \mathrm{g}$ and $4^{\circ} \mathrm{C}$ for $15 \mathrm{~min}$. Western blotting was performed as described previously.

Statistical analysis. Statistical analyses were performed using SPSS 17.0 (SPSS, Inc., Chicago, IL, USA). Student's t-test or Pearson's chi-squared tests were performed as appropriate. Analysis of variance (ANOVA) was used for repeated measurements (one-way ANOVA or Kruskal-Wallis test). $\mathrm{P}<0.05$ was considered to indicate a statistically significant difference.

\section{Results}

Src and p-Src expression in ovarian cancer cell lines. Src and p-Src expression levels were measured using western blotting. The six ovarian cancer cell lines exhibited high levels of Src and p-Src expression. A2780 and SKOV3 cells lines demonstrated the highest Src expression levels, whilst the A2780 and HO8910 cell lines demonstrated the highest p-Src expression levels (Fig. 1). Based on these results, the A2780 and HO8910 cell lines were selected for further analysis.

Paclitaxel activates the Src pathway in ovarian cancer cells. p-Src expression was upregulated in a dose-dependent manner. However, total Src protein expression was unaffected, in A2780 and HO8910 cell lines, following treatment with paclitaxel. For the A2780 cells, p-Src expression was greatest following $24 \mathrm{~h}$ of treatment with $10 \mathrm{nmol} / 1$ paclitaxel. For HO8910 cells, p-Src expression was greatest following $6 \mathrm{~h}$ of treatment with $5 \mathrm{nmol} / \mathrm{l}$ (Fig. 2).

Dasatinib inhibits viability and enhances paclitaxel cytotoxicity in ovarian cancer cells. The results of the present study demonstrated that paclitaxel treatment activated Src expression in ovarian cancer cells. Subsequently, the effect of dasatinib on the cytotoxicity of paclitaxel was investigated in these cell lines. A2780 and HO8910 cell lines treated with increasing dasatinib concentrations $(0.3125-20 \mu \mathrm{M})$ demonstrated a dose-dependent reduction in cell viability (Fig. 3A). $\mathrm{IC}_{50}$ values 
A

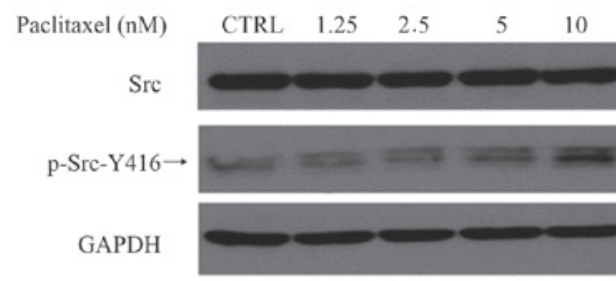

B

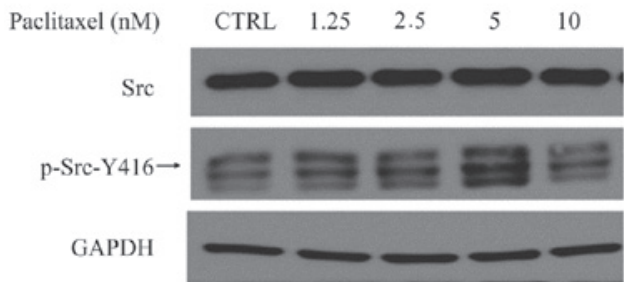

A2780

Time (h)

$\operatorname{Src}$

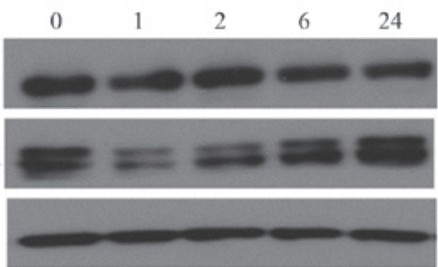

GAPDH

HO8910

Time (h)

$\operatorname{Src}$

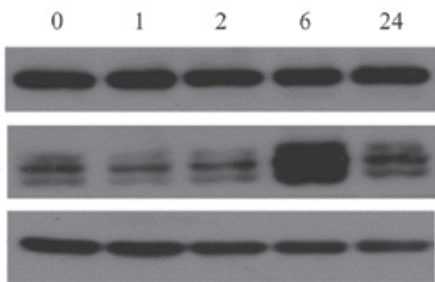

Figure 2. Src expression and phosphorylation in A2780 and HO8910 cell lines. (A) A2780 cells treated with different concentrations of paclitaxel for $6 \mathrm{~h}$ (left) and $10 \mathrm{nM}$ paclitaxel for different times (right); (B) HO8910 cells treated with different concentrations paclitaxel for $6 \mathrm{~h}$ (left) and $10 \mathrm{nM}$ paclitaxel for different times (right). CTRL, control; p-Src, phospho-Src-Y416.

$\mathbf{A}$

a

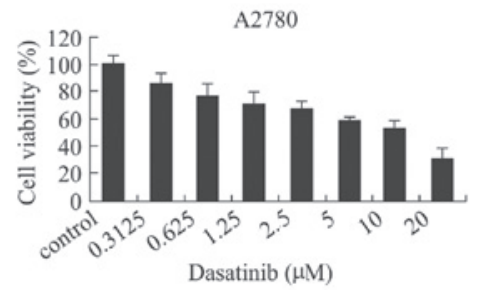

b

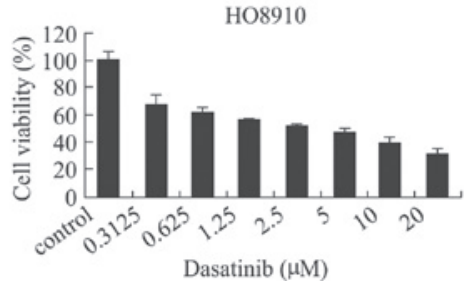

B

a

A2780

b

HO8910

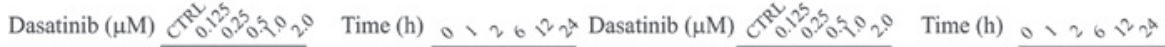

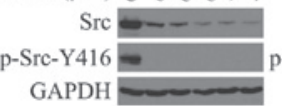

$\mathrm{Src}$ -Src-Y416
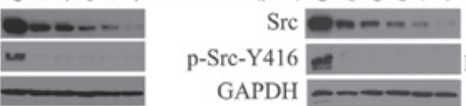
p-Src-Y416 GAPDH

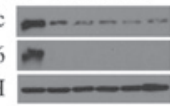

C

a

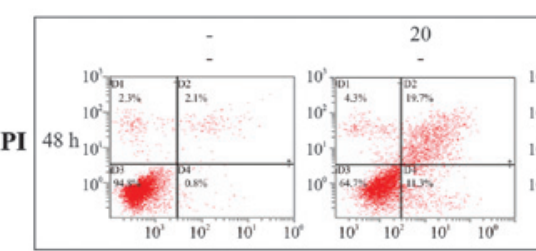

A2780
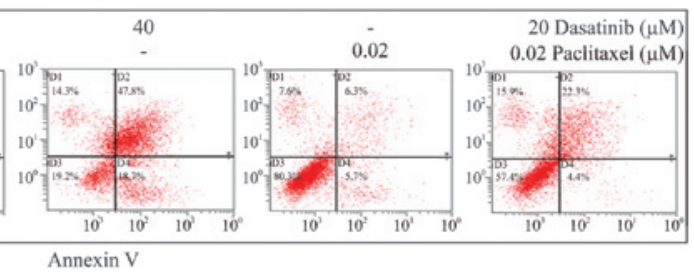

b

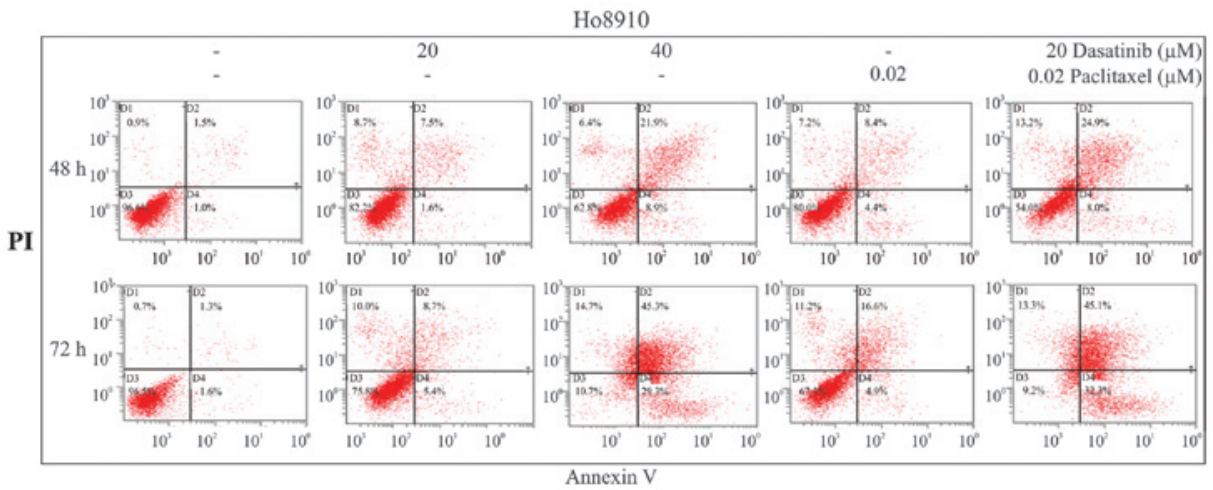

Figure 3. Dasatinib enhanced the paclitaxel-associated cytotoxicity in ovarian cancer cells. (A) Cell death following treatment with dasatinib for $72 \mathrm{~h}$, in A2780 and HO8910 cell lines. Cell death was determined using an MTT assay. The $\mathrm{IC}_{50}$ of dasatinib was $7.30 \pm 0.64 \mu \mathrm{M}$ in A2780 cells and $2.51 \pm 0.64 \mu \mathrm{M}$ in $\mathrm{HO} 8910$ cells. (B) Src expression and phosphorylation in A2780 and HO8910 cell lines. (Ba) Left, A2780 cells treated with dasatinib for $2 \mathrm{~h}$; right, A2780 cells treated with $0.1 \mu \mathrm{M}$ dasatinib over time. (Bb) Left, HO8910 cells treated with dasatinib for $2 \mathrm{~h}$; right, HO8910 cells treated with $0.1 \mu \mathrm{M}$ dasatinib over time. Control cells were treated with $0.02 \%$ dimethyl sulfoxide. (C) Dasatinib enhanced cell apoptosis and enhanced paclitaxel-induced cytotoxicity in ovarian cancer cells. (Ca) A2780 cells treated with 20 and $40 \mu \mathrm{M}$ dasatinib for $48 \mathrm{~h}$ exhibited apoptosis rates of $30.9 \pm 1.4$ and $66.5 \pm 0.9 \%$ of the control, respectively. (Cb) HO8910 cells treated with 20 and $40 \mu \mathrm{M}$ dasatinib for $48 \mathrm{~h}$ exhibited apoptosis rates of $8.65 \pm 0.6$ and $30.8 \pm 1.2 \%$ of the control, respectively. At $72 \mathrm{~h}$ the rates were $12.6 \pm 2.2$ and $76.0 \pm 2.0 \%$ of the controls, respectively. $(\mathrm{P}<0.05)$. p-Src, phospho-Src-Y416. 
Table I. Effects of dasatinib combined with paclitaxel.

A, A2780 ovarian cancer cell line

\begin{tabular}{lccr}
\hline Dasatinib $(\mu \mathrm{M})$ & Paclitaxel $(\mu \mathrm{M})$ & Fa & CI \\
\hline 0.3125 & 0.0013 & 0.4089 & 0.330 \\
0.6250 & 0.0025 & 0.4247 & 0.575 \\
1.2500 & 0.0050 & 0.4650 & 0.817 \\
2.5000 & 0.0100 & 0.5277 & 0.973 \\
5.0000 & 0.0200 & 0.6903 & 0.483 \\
10.000 & 0.0400 & 0.8178 & 0.245 \\
\hline
\end{tabular}

B, HO8910 ovarian cancer cell line

\begin{tabular}{lccr}
\hline Dasatinib $(\mu \mathrm{M})$ & Paclitaxel $(\mu \mathrm{M})$ & Fa & CI \\
\hline 0.3125 & 0.0006 & 0.4713 & 0.402 \\
0.625 & 0.0013 & 0.5130 & 0.526 \\
1.25 & 0.0025 & 0.5500 & 0.746 \\
2.5 & 0.0050 & 0.7395 & 0.308 \\
5.0 & 0.0100 & 0.8294 & 0.257 \\
10.0 & 0.0200 & 0.8229 & 0.553 \\
\hline
\end{tabular}

CI, confidence interval; $\mathrm{Fa}$, inhibition rate.

were $7.30 \pm 0.64 \mu \mathrm{M}$ in $\mathrm{A} 2780$ cells and $2.51 \pm 0.64 \mu \mathrm{M}$ in HO8910 cells. Cell lines were sensitive to paclitaxel treatment $\left(\mathrm{IC}_{50} \sim 20 \mathrm{nmol} / \mathrm{l}\right)$. Dasatinib combined with paclitaxel demonstrated synergistic antitumor activity with confidence interval (CI) values of 0.25-0.93 and 0.31-0.75 in A2780 and HO8910 cell lines, respectively (Table I). In addition, at concentrations below the $\mathrm{IC}_{50}$, dasatinib suppressed $\mathrm{Src}$ and $\mathrm{p}$-Src protein expression (Fig. 3B). An annexin V-FITC/propidium iodide apoptosis assay was conducted in order to determine whether the cytotoxicity of dasatinib, with or without paclitaxel treatment, is associated with changes in cell apoptosis. Apoptosis rates in A2780 cells treated with $20 \mu \mathrm{M}$ and $40 \mu \mathrm{M}$ dasatinib for $48 \mathrm{~h}$, were $30.9 \pm 1.4$ and $66.5 \pm 0.9 \%$, respectively. In HO8910 cells, these rates were $8.65 \pm 0.6$ and $30.8 \pm 1.2 \%$ following $48 \mathrm{~h}$ of treatment, and $12.6 \pm 2.2$ and $76.0 \pm 2.0 \%$ following $72 \mathrm{~h}$ of treatment, respectively $(\mathrm{P}<0.05)$. A greater percentage of apoptotic cells was observed following treatment with dasatinib combined with paclitaxel, compared with cell treated with dasatinib only. The apoptotic rates of A2780 cells following treatment with $20 \mu \mathrm{M}$ dasatinib, $0.02 \mu \mathrm{M}$ paclitaxel, and combined dasatinib and paclitaxel, for $48 \mathrm{~h}$, were $34.7 \pm 3.4,20.9 \pm 3.7$ and $44.2 \pm 2.4 \%$, respectively. Apoptotic rates were 17.3 $\pm 2.1,22.0 \pm 2.7$ and $43.6 \pm 4.0 \%$ in $\mathrm{HO} 8910$ cells (all $\mathrm{P}<0.001$ compared with control cells; Fig. 3C).

Antitumor activity of combined dasatinib and paclitaxel in a human ovarian cancer xenograft model. A2780 and HO8910 xenografts were established in nude mice in order to assess the potential antitumor effect of treatment with dasatinib alone or with dasatinib in combination with paclitaxel. In A2780 and HO8910 xenografts, dasatinib treatment led to tumor growth inhibition by 43.2\% (A2780) and 34.0\% (HO8910; Table II; Fig. 4A). Combined treatment led to a greater growth inhibitory effect: $\mathrm{TIR}_{\mathrm{A} 2780}=76.7 \%(\mathrm{P}<0.001)$ and $\mathrm{TIR}_{\mathrm{HO} 8910}=58.5 \%(\mathrm{P}<0.001)$ in comparison with single drug treatments. The comparison of tumor-growth curves, using group and time as variables, with a two-sided ANOVA, demonstrated that the group-by-time interaction for tumor growth $(\mathrm{F})$ was statistically significant $\left(\mathrm{F}_{\mathrm{A} 2780}=8.054, \mathrm{P}_{\mathrm{A} 2780}<0.001\right.$; $\left.\mathrm{F}_{\mathrm{H} 08910}=7.681, \mathrm{P}_{\mathrm{HO8910}}<0.001\right)$. As shown in Table II, mouse weight was not significantly different between groups (one-way ANOVA: $\mathrm{F}_{\mathrm{A} 2780}=5.619, \mathrm{P}_{\mathrm{A} 2780}=0.162 ; \mathrm{F}_{\mathrm{HO} 8910}=0.6$, $\left.\mathrm{P}_{\text {HO8910 }}=0.794\right)$. Overall, treatments appeared to be relatively well tolerated by the mice, with no mortalities or weight loss, and no signs of acute or delayed toxicity. Western blotting (Fig. 4B) and immunohistochemistry (Fig. 4C) suggested that paclitaxel activated and upregulated the Src pathway ( $\mathrm{p}$-Src expression) in cancer cells, compared with that in the control cells. Dasatinib combined with paclitaxel treatment downregulated p-Src expression in cancer cells (23.3 vs. $31.0 \%$ in A2780 cells and 21.3 vs. $48.7 \%$ in HO8910 cells), as compared with dasatinib treatment alone (Figs. 4B and C). This is consistent with the results of the in vitro study.

\section{Discussion}

Ovarian carcinoma is the leading cause of reproductive-associated cancer mortality among females worldwide (1). Despite high initial remission rates using paclitaxel and platinum-based chemotherapy, $<75 \%$ of patients with advanced-stage ovarian carcinoma will relapse (1). Targeted agents have been the primary focus of treatment efforts for patients with recurrent 
Table II. Body weight, tumor weight and tumor volume in ovarian cancer xenografts.

A, A2780 xenografts

\begin{tabular}{|c|c|c|c|c|c|c|}
\hline \multirow[b]{2}{*}{ Treatment } & \multirow[b]{2}{*}{$\mathrm{n}$} & \multicolumn{2}{|c|}{ Weight (g) } & \multirow[b]{2}{*}{ Tumor volume } & \multirow[b]{2}{*}{ Tumor weight } & \multirow[b]{2}{*}{ TIR } \\
\hline & & Day 0 & Day 31 & & & \\
\hline Control & $5 / 8$ & $20.88 \pm 0.18$ & $22.47 \pm 0.89$ & $2465.0 \pm 459.1$ & $2.12 \pm 0.34$ & \\
\hline Dasatinib & $6 / 12$ & $20.93 \pm 0.72$ & $22.37 \pm 0.89$ & $1400.2 \pm 231.3$ & $1.67 \pm 0.30$ & 43.197 \\
\hline Paclitaxel & $6 / 11$ & $21.17 \pm 0.45$ & $23.08 \pm 0.72$ & $1016.9 \pm 176.3$ & $1.34 \pm 0.20$ & 58.764 \\
\hline Combined & $6 / 11$ & $21.25 \pm 0.41$ & $21.7 \pm 1.1$ & $574.3 \pm 126.1$ & $0.93 \pm 0.19$ & 76.702 \\
\hline
\end{tabular}

B, HO8910 xenografts

\begin{tabular}{llccrrr}
\hline & & \multicolumn{2}{c}{ Weight $(\mathrm{g})$} & & & \\
\cline { 3 - 4 } Treatment & $\mathrm{n}$ & Day 0 & Day 31 & & Tumor volume & Tumor weight \\
\hline Control & $6 / 11$ & $18.30 \pm 0.60$ & $22.32 \pm 0.40$ & & $1695.2 \pm 162.5$ & $2.65 \pm 0.28$ \\
Dasatinib & $6 / 10$ & $19.10 \pm 0.66$ & $20.88 \pm 0.58$ & & $1119 \pm 168.6$ & $1.55 \pm 0.30$ \\
Paclitaxel & $6 / 12$ & $19.45 \pm 0.66$ & $20.57 \pm 0.88$ & & $923.2 \pm 139.9$ & $1.54 \pm 0.22$ \\
Combined & $6 / 10$ & $17.28 \pm 0.51$ & $20.35 \pm 0.57$ & & $698.3 \pm 145.7$ & $1.65 \pm 0.42$ \\
\hline
\end{tabular}

Tumor volume $\left(\mathrm{mm}^{3}\right)$; tumor weight $(\mathrm{g})$; TIR $(\%)$. Data are presented as the mean \pm standard deviation. TIR, tumor growth inhibitory rates.

ovarian cancer. A number of studies have aimed to investigate novel antitumor drugs that interfere with the critical signaling pathways associated with the occurrence and progression of ovarian cancer. Dasatinib is a competitive inhibitor of a number of tyrosine kinases, including SFKs (27). A number of studies have suggested that dasatinib alone or in combination with other drugs, such as paclitaxel, exhibits preclinical antitumor activity in human ovarian cancer $(22,23,25)$. However, the synergistic mechanisms associated with dasatinib treatment combined with paclitaxel, remain unknown. The results of the present study suggested that dasatinib treatment enhanced the paclitaxel-associated antitumor activities in ovarian cancer cells. This may be explained by the inhibitory effects of dasatinib on the Src signal pathway.

Mayer and Krop (28) demonstrated that Src regulates a number of signaling pathways that affect proliferation, survival, migration, invasion and angiogenesis in tumor cells, and that Src is activated and overexpressed in numerous types of solid tumors $(6-10,16,26,29)$. The current study demonstrated overexpression of Src and $\mathrm{p}-\mathrm{Src}$ in human ovarian cancer tissues (13). In the present study, the Src signaling pathway was activated, and p-Src was overexpressed in six human ovarian cancer cell lines. Given the effect of Src signaling on ovarian cancer, Konecny et al (22) studied 34 ovarian cancer cell lines and demonstrated that dasatinib inhibits cell proliferation in vitro with $0.001-11.3 \mu \mathrm{M} \mathrm{IC}{ }_{50}$ values. In the current study, dasatinib was found to inhibit ovarian cancer cell proliferation. Low concentration dasatinib $\left(\mathrm{IC}_{50} \sim 8 \mu \mathrm{M}\right)$ reduced cell proliferation, and Src and $\mathrm{p}-\mathrm{Src}$ protein expression levels were downregulated in response to dasatinib treatment at varying concentrations and incubation times. Therefore, dasatinib cytotoxicity in ovarian cancer may be explained by the inhibition of Src pathway activation. Teoh et al (23) suggested that dasatinib caused a reduction in $\mathrm{p}$-Src expression in ovarian cell lines. The results of the present study confirm these findings. Furthermore, the results of the current study suggested that paclitaxel activated Src signaling in ovarian cancer cells. In the current study, p-Src expression was upregulated in A2780 and HO8910 cell lines following treatment with different concentrations of paclitaxel. However, Src protein expression was not upregulated. These results were confirmed in the in vivo studies. Based on the results of the present study, it is hypothesized that primary and acquired resistance to paclitaxel in ovarian cancer may be due to the activation of the Src signaling pathway, and the associated cancer cell proliferation, angiogenesis, invasion and dissemination.

A significant synergetic antitumor effect was observed following treatment with a combination of dasatinib and paclitaxel, which was associated with inhibition of Src pathway activity. In accordance with the results of the in vitro study, dasatinib suppressed the growth of ovarian cancer xenografts in nude mice compared with the controls (TIR 43.2\% in A2780 cells, and $34.0 \%$ in HO8910 cells, both $\mathrm{P}<0.01)$. Furthermore, when combined with paclitaxel, a synergistic antitumor effect was observed in vitro $(\mathrm{CI}<1$; TIR values were 76.7 and $58.5 \%$ in A2780 cells and HO8910 cells, respectively, $\mathrm{P}<0.05)$. The synergy between dasatinib and paclitaxel was associated with the inhibition of $\mathrm{p}$-Src protein expression. These results suggested that paclitaxel exhibits antitumor activity in ovarian cancer, but may also activate the Src signal pathway, which promotes cell survival and drug resistance.

The mechanisms underlying dasatinib-enhanced antitumor activity and the association with paclitaxel, remain unclear. 
A

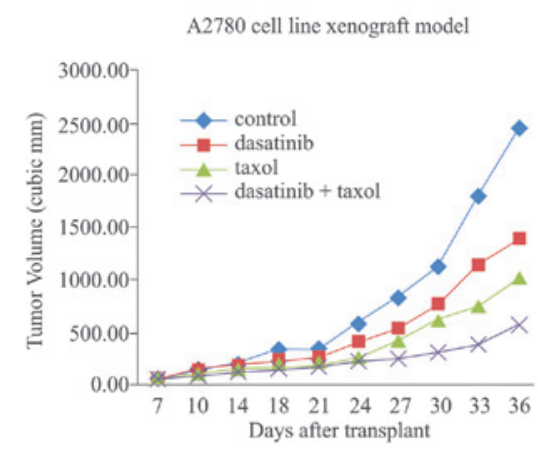

B
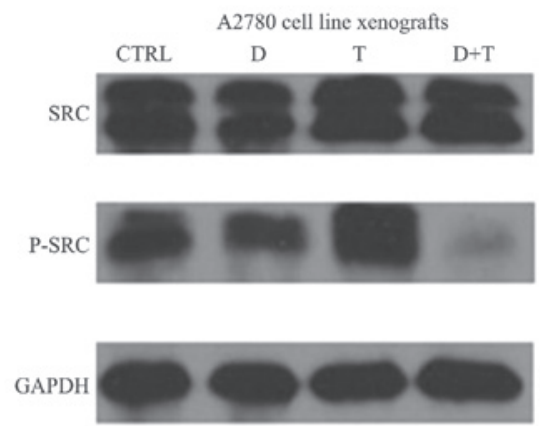

C

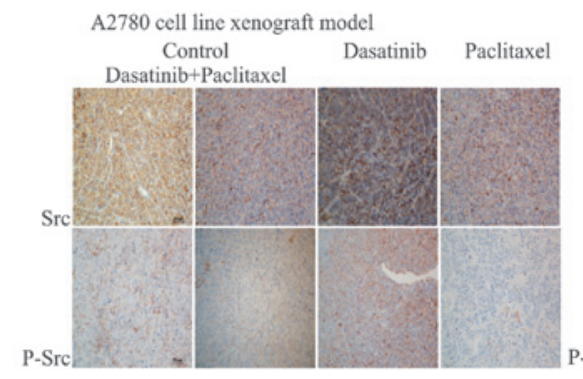

GAPDH
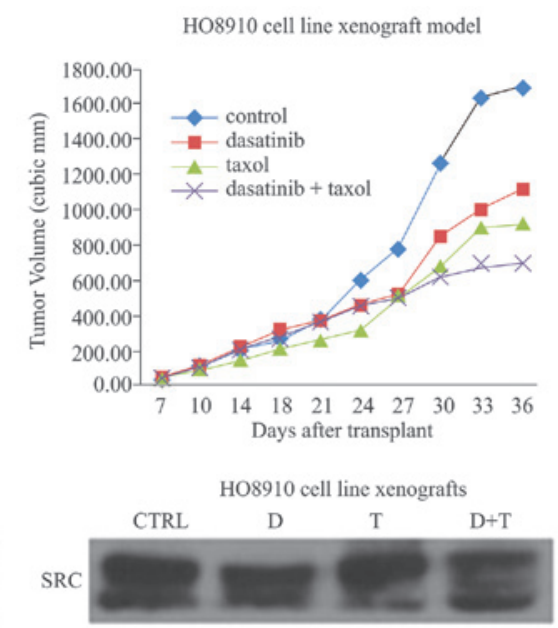

P-SRC
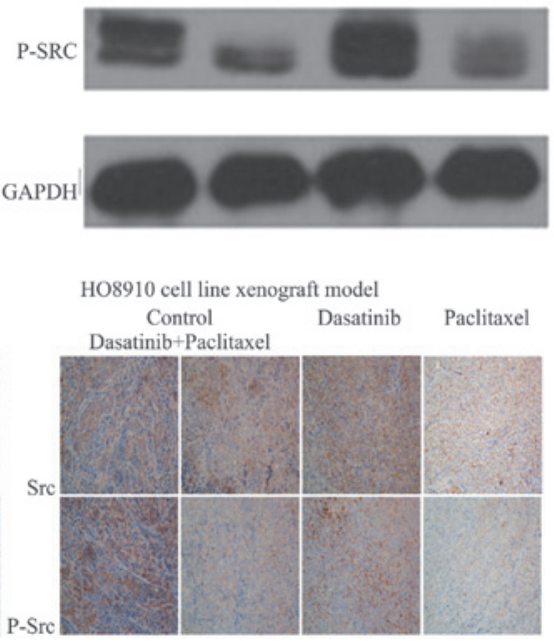

Figure 4. Antitumor activity of combined dasatinib and paclitaxel in a human ovarian cancer xenograft model. (A) Ovarian cancer xenograft growth curve. Mice bearing ovarian cancer xenografts were treated as follows: Dasatinib alone (10 mg/kg five times a week); paclitaxel alone (15 mg/kg once a week); combination of dasatinib (10 mg/kg per week); and paclitaxel $(15 \mathrm{mg} / \mathrm{kg}$ per week); vehicle control (the same quantity of sterile distilled water). P=0.003, dasatinib vs. control in A2780 cells; $\mathrm{P}=0.004$, combined treatment vs. dasatinib alone in A2780 cells; $\mathrm{P}=0.087$, combined treatment vs. paclitaxel in A2780 cells; $\mathrm{P}=0.024$, dasatinib vs. control in HO8910 cells; $\mathrm{P}=0.075$, combined treatment vs. dasatinib alone in HO8910 cells; $\mathrm{P}=0.281$, combined treatment vs. paclitaxel in HO8910 cells. Group-by-time interaction for tumor growth was statistically significant (A2780, F=8.054 and P<0.001; HO8910, F=7.681 and P<0.001). (B) Western blot analysis of Src and p-Src protein in vivo. (C) Immunohistochemistry of Src and p-Src protein expression in ovarian cancer xenografts. p-Src, phospho-Src-Y416; CTR, control; D, dasatinib; T, paclitaxel.

SFK inhibitors enhance paclitaxel sensitivity by inducing cell apoptosis, autophagy, microtubule stability and neovasculature (30). George et al (31) demonstrated that Src inhibition reduces the critical intracellular concentration at which paclitaxel induces tubulin stabilization and apoptosis. Furthermore, Chen et al (32) demonstrated that Src inhibition alone activates caspase- 3 and promotes apoptosis. The results of the present study suggested that dasatinib promoted apoptosis in a mouse xenograft model. The results suggested that dasatinib exhibited a dose- and time-dependent effect on the level of apoptosis in ovarian cancer cell lines (both $\mathrm{P}<0.05$ ). Therefore, dasatinib is capable of enhancing apoptosis following paclitaxel treatment.

The use of additive targets in cancer therapy, such as the use of targeted agents in combination with cytotoxic drugs, is becoming increasingly common. Ovarian cancer is highly responsive to first-line chemotherapy, following optimal debulking surgery. However, relapses are frequently observed in patients, due to the development of resistance to conventional chemotherapy drugs. Therefore, combination therapy may be useful for the treatment of ovarian cancer (33). Novel therapeutic agents in combination with conventional therapies may prevent the emergence of resistance, thereby prolonging remission and improving long-term survival. In the present study, a synergistic effect was observed following treatment with a combination of dasatinib and paclitaxel, in vitro and in vivo. A number of studies have demonstrated that SFK inhibition sensitizes cancer cells to paclitaxel-based treatments by modulating cell survival and proliferation, autophagy, microtubule stability, motility, and tumor angiogenesis $(23,32,34-36)$. Numerous mechanisms have been proposed in order to explain these observations.

The present study demonstrated that $\mathrm{p}$-Src expression levels were markedly increased following paclitaxel treatment. Therefore, paclitaxel activates Src signaling. However, dasatinib treatment appeared to suppress p-Src expression, in vitro and in vivo. Similarly, a separate study demonstrated that oxaliplatin activates Src signaling via a reactive oxygen species-dependent mechanism, and a trend was observed between the degree of Src activation following oxaliplatin treatment and the degree of 
synergy between dasatinib and oxaliplatin (37). Furthermore, acquired and persistent resistance to paclitaxel is associated with upregulation of Multi-Drug Resistance (MDR)-1,increased DNA damage tolerance and drug metabolism, altered microtubule isotype expression and mutations of $\beta$-tubulin (31). The inhibition of certain SFKs eliminated the resistance of ovarian cancer cells via an MDR-independent mechanism. However, the pathways underlying this process remain unclear (31). A study has shown that dasatinib may enhance paclitaxel sensitivity by suppressing B cell lymphoma-2 and cyclin dependent kinase 1 expression in ovarian cancer cells via a p27(Kip1)-dependent process (34). The mechanisms involved in the paclitaxel-activated Src pathway are unclear. However, it is clear that this activation is a primary factor in the synergistic effects of combined dasatinib and paclitaxel treatment in ovarian cancer. The present study suggested that dasatinib increased the cytotoxicity of paclitaxel in ovarian cancer, possibly a result of dasatinib-driven inhibition of paclitaxel-induced Src activity. Therefore, in response to dasatinib treatment, apoptosis was enhanced and paclitaxel resistance was delayed in ovarian cancer cells.

The preclinical data in the present study suggests that dasatinib is a potential therapeutic agent for ovarian cancer. When combined with paclitaxel, dasatinib is able to further enhance anti-ovarian cancer activity, and may delay or prevent paclitaxel resistance.

\section{Acknowledgements}

The authors would like to thank Professor Xiaofeng Zhu for his support in conducting this study. The study was funded by The Natural Science Foundation of Guangdong Province (S2013010015575).

\section{References}

1. Jelovac D and Armstrong DK: Recent progress in the diagnosis and treatment of ovarian cancer. CA Cancer J Clin 61: 183-203, 2011.

2. Siegel R, Naishadham D and Jemal A: Cancer statistics, 2012. CA Cancer J Clin 62: 10-29, 2012.

3. Shah NP, Tran C, Lee FY, Chen P, Norris D and Sawyers CL: Overriding imatinib resistance with a novel ABL kinase inhibitor Science 305: 399-401, 2004.

4. Yeatman TJ: A renaissance for SRC. Nat Rev Cancer 4 470-480, 2004

5. Brunton VG and Frame MC: Src and focal adhesion kinase as therapeutic targets in cancer. Curr Opin Pharmacol 8: 427-432, 2008.

6. Wheeler DL, Iida M and Dunn EF: The role of Src in solid tumors. Oncologist 14: 667-678, 2009.

7. Summy JM and Gallick GE: Src family kinases in tumor progression and metastasis. Cancer Metastasis Rev 22: 337-358, 2003.

8. Dehm SM and Bonham K: SRC gene expression in human cancer: The role of transcriptional activation. Biochem Cell Biol 82: 263-274, 2004.

9. Morgan L, Nicholson RI and Hiscox S: SRC as a therapeutic target in breast cancer. Endocr Metab Immune Disord Drug Targets 8: 273-278, 2008.

10. Lutz MP, Esser IB, Flossmann-Kast BBM, et al: Overexpression and activation of the tyrosine kinase Src in human pancreatic carcinoma. Biochem Biophys Res Commun 243: 503-508, 1998.

11. Budde RJ, Ke S and Levin VA: Activity of pp60c-src in 60 different cell lines derived from human tumors. Cancer Biochem Biophys 14: 171-175, 1994.

12. Wiener JR, Windham TC, Estrella VC, et al: Activated SRC protein tyrosine kinase is overexpressed in late-stage human ovarian cancers. Gynecol Oncol 88: 73-79, 2003.

13. Huang YW, Chen C, Xu MM, Li JD, Xiao J and Zhu XF: Expression of c-Src and phospho-Src in epithelial ovarian carcinoma. Mol Cell Biochem 376: 73-79, 2013.
14. Lombardo LJ, Lee FY, Chen $\mathrm{P}$, et al: Discovery of N-(2-chloro-6-methyl-phenyl)-2-(6-(4-(2-hydroxyethyl)-piperazin -1-yl)-2-methylpyrimidin-4-ylamino)thiazole-5-carboxamide(BMS354825), a dual Src/Abl kinase inhibitor with potent antitumor activity in preclinical assays. J Med Chem 47: 6658-6661, 2004.

15. Gnoni A, Marech I, Silvestris N, Vacca A and Lorusso V: Dasatinib: an anti-tumour agent via Src inhibition. Curr Drug Targets 12: 563-578, 2011.

16. Nam S, Kim D, Cheng JQ, et al: Action of the Src family kinase inhibitor, dasatinib (BMS-354825), on human prostate cancer cells. Cancer Res 65: 9185-9189, 2005.

17. Johnson FM, Saigal B, Talpaz M and Donato NJ: Dasatinib (BMS-354825) tyrosine kinase inhibitor suppresses invasion and induces cell cycle arrest and apoptosis of head and neck squamous cell carcinoma and non-small cell lung cancer cells. Clin Cancer Res 11: 6924-6932, 2005.

18. Ceppi P, Papotti M, Monica V, et al: Effects of Src kinase inhibition induced by dasatinib in non-small cell lung cancer cell lines treated with cisplatin. Mol Cancer Ther 8: 3066-3074, 2009.

19. Tsao AS, He D, Saigal B, et al: Inhibition of c-Src expression and activation in malignant pleural mesothelioma tissues leads to apoptosis, cell cycle arrest, and decreased migration and invasion. Mol Cancer Ther 6: 1962-1972, 2007.

20. Trevino JG, Summy JM, Lesslie DP, et al: Inhibition of SRC expression and activity inhibits tumor progression and metastasis of human pancreatic adenocarcinoma cells in an orthotopic nude mouse model. Am J Pathol 168: 962-972, 2006.

21. Shor AC, Keschman EA, Lee FY, et al: Dasatinib inhibits migration and invasion in diverse human sarcoma cell lines and induces apoptosis in bone sarcoma cells dependent on SRC kinase for survival. Cancer Res 67: 2800-2808, 2007.

22. Konecny GE, Glas R, Dering J, et al: Activity of the multikinase inhibitor dasatinib against ovarian cancer cells. Br J Cancer 101: 1699-1708, 2009

23. Teoh D, Ayeni TA, Rubatt JM, Adams DJ, Grace L and Starr MD: Dasatinib (BMS-35482) has synergistic activity with paclitaxel and carboplatin in ovarian cancer cells. Gynecol Oncol 121: 187-192, 2011.

24. Deng R, Tang J, Xia LP, et al: ExcisaninA, a diterpenoid compound purified from Isodon MacrocalyxinD, induces tumor cells apoptosis and suppresses tumor growth through inhibition of PKB/AKT kinase activity and blockade of its signal pathway. Mol Cancer Ther 8: 873-882, 2009.

25. Le XF, Mao W, Lu Z, Carter BZ and Bast RC Jr: Dasatinib induces autophagic cell death in human ovarian cancer. Cancer 116: 4980-4990, 2010.

26. Vitali R, Mancini C, Cesi V, et al: Activity of tyrosine kinase inhibitor Dasatinib in neuroblastoma cells in vitro and in orthotopic mouse model. Int J Cancer 125: 2547-2555, 2009.

27. Kim LC, Rix U and Haura EB: Dasatinib in solid tumors. Expert Opin Investig Drugs 19: 415-425, 2010.

28. Mayer EL and Krop IE: Advances in targeting SRC in the treatment of breast cancer and other solid malignancies. Clin Cancer Res 16: 3526-3532, 2010.

29. Yezhelyev MV, Koehl G, Guba M, et al: Inhibition of SRC tyrosine kinase as treatment for human pancreatic cancer growing orthotopically in nude mice. Clin Cancer Res 10: 8028-8036, 2004.

30. Le XF and Bast RC Jr: Src family kinases and paclitaxel sensitivity. Cancer Biol Ther 12: 260-269, 2011.

31. George JA, Chen T and Taylor CC: SRC tyrosine kinase and multidrug resistance protein-1 inhibitions act independently but cooperatively to restore paclitaxel sensitivity to paclitaxel-resistant ovarian cancer cells. Cancer Res 65: 10381-10388, 2005.

32. Chen T, Pengetnze Y and Taylor CC: Src inhibition enhances paclitaxel cytotoxicity in ovarian cancer cells by caspase-9-independent activation of caspase-3. Mol Cancer Ther 4: 217-224, 2005.

33. Agarwal R and Kaye SB: Ovarian cancer: strategies for overcoming resistance to chemotherapy. Nat Rev Cancer 3: 502-516, 2003.

34. Le XF, Mao W, He G, et al: The role of p27(Kip1) in dasatinib-enhanced paclitaxel cytotoxicity in human ovarian cancer cells. J Natl Cancer Inst 103: 1403-1422, 2011.

35. Han LY, Landen CN, Trevino JG, et al: Antiangiogenic and antitumor effects of SRC inhibition in ovarian carcinoma. Cancer Res 66: 8633-8639, 2006.

36. Kim HS, Han HD, Armaiz-Pena GN, et al: Functional roles of Src and Fgr in ovarian carcinoma. Clin Cancer Res 17: 1713-1721, 2011.

37. Kopetz S, Lesslie DP, Dallas NA, et al: Synergistic activity of the SRC family kinase inhibitor dasatinib and oxaliplatin in colon carcinoma cells is mediated by oxidative stress. Cancer Res 69: 3842-3849, 2009. 